\title{
Métodos de identificação de zonas de acumulação de acidentes: Revisão e aplicação a um caso de estudo
}

\author{
Sara Ferreira ${ }^{1}$, Joana Martins ${ }^{2}$
}

\begin{abstract}
Resumo: Neste trabalho apresenta-se uma descrição e análise resumida bem como a aplicação dos vários métodos de identificação de zonas de acumulação de acidentes propostos pelo manual Highway Safety Manual (HSM). É reconhecido internacionalmente a importância deste manual e a sua utilização pelos profissionais responsáveis da segurança viária. $\mathrm{O}$ HSM propõe e descreve 13 métodos para identificação de zonas de acumulação de acidentes, todos muito distintos quer em termos de âmbito de aplicação quer na complexidade dos mesmos. Com o objetivo de simplificar a escolha e aplicação destes métodos, o presente estudo descreve e organiza os diferentes métodos segundo 3 critérios que podem ser decisivos na escolha e aplicação dos métodos. Para além dos 13 métodos do HSM, apresenta-se ainda um método desenvolvido recentemente e designado de modelo binário probit. Para analisar os referidos métodos, considerou-se como caso de estudo a cidade do Porto, Portugal. Considerando este caso de estudo, foi possível analisar as dificuldades e especificidades de cada método, tendo-se verificado que nem todos os métodos foram possíveis de se aplicar. Os resultados da aplicação dos métodos foram analisados e comparados com base em testes de avaliação de desempenho. Os testes indicam que os métodos excesso previsto da frequência média de acidentes proposto pelo referido manual e o modelo binário probit, mais recentemente desenvolvido, apresentam os melhores resultados. Os resultados desta aplicação poderão servir de base para futuras aplicações noutras regiões e/ou países.
\end{abstract}

Palavras-chave: Highway Safety Manual. Segurança viária. Zonas de acumulação de acidentes. Medidas de desempenho. Interseções.

Abstract: This paper presents a description of the various methods proposed by the Highway Safety Manual (HSM) to identify hotspots. In fact, this manual has become a tool for the professional in road safety all over the world. The HSM suggest and described 13 distinct methods in terms of application context and method complexity. Therefore, the main goal of this paper is to describe and organize those methods to help safety professionals to select the method(s) to apply to the study case. Thus, the various methods are organized based on 3 application criteria, which may be crucial to the decision process. In addition to the HSM methods, a recent method entitled probit binary model is also shown. An application to the Porto city is presented in order to better analyze each method. In fact, based on this study case, it was concluded that not all of the methods were possible to apply to this study case. A comparison of the various methods is developed using tests to assess the performance of each method. The methods named excess predicted average crash frequency proposed by the manual and the binary probit model, recently developed, present the best results based on those tests. The results of the case study application may be the basis for future applications in other regions and/or countries.

Keywords: Highway Safety Manual. Road safety. Hotspot. Performance measure. Intersections.

\section{INTRODUÇÃO}

A identificação e tratamento de zonas de acumulação de acidentes (ZAA), vulgarmente designada de ponto negro (“hot spots" na terminologia inglesa), é um passo reconhecido por técnicos especialistas e investigadores da área como fundamental para melhorar o desempenho de uma rede viária em termos de segurança ( Cheng e Washington, 2008). De facto, a aplicação de medidas de tratamento a locais identificados como ZAA tem demonstrado resultar numa diminuição significativa do número de acidentes, muitas vezes associada a baixos custos em termos de investimento.

\footnotetext{
1 Faculdade de Engenharia da Universidade do Porto, Porto, Portugal (sara@fe.up.pt).

2 Faculdade de Engenharia da Universidade do Porto, Porto, Portugal (ec07199@fe.up.pt).
}

Manuscrito recebido em 30/06/2014 e aprovado para publicação em 24/09/2014. Este artigo é parte de TRANSPORTES v. 22, n. 3, 2014. ISSN: 2237-1346 (online).

DOI: http://dx.doi.org/10.14295/transportes.v22i3.813.
Diversos trabalhos têm vindo a ser desenvolvidos e aplicados neste âmbito, nomeadamente estudos que analisam métodos de identificação de ZAA (MIZAA) para seleção de locais a tratar (Kononov, 2002, Geurts, et al., 2004, Hauer, et al., 2004, Geedipally e Lord, 2010, Bandyopadhyaya e Mitra, 2011, Cafiso e Silvestro, 2011, Coll, et al., 2013). Cada método é descrito em contextos e enquadramentos distintos, o que não permite facilmente comparar e até mesmo caracterizar cada MIZAA.

Recentemente, o documento designado de Highway Safety Manual (HSM) (AASHTO, 2010) compilou alguns desses métodos de identificação de ZAA. Esses MIZAA são descritos na Parte B do referido manual (o HSM é composto por 4 partes), na qual são apresentados diversos passos que podem ser utilizados para monitorizar e reduzir a frequência dos acidentes e sua gravidade. Mais especificamente, a Parte B do HSM apresenta um processo de ges- 
tão de segurança rodoviária (Roadway Safety Management Process), organizado em 6 capítulos: Rastreamento da Rede "Network Screening"; Diagnóstico "Diagnosis"; Seleção das Medidas de Tratamento "Select Countermeasures"; Avaliação Económica "Economic Appraisal", Prioritização de Projetos "Prioritize Projects"; Avaliação da Eficiência em termos de Segurança "Safety Effectiveness Evaluation". O capítulo 4, Rastreamento da Rede "Network Screening", propõe um procedimento de análise de uma rede de transportes com o objetivo de identificar e classificar os locais de acordo com o potencial de reduzir a frequência de acidentes através da implementação de medidas de tratamento.

O procedimento proposto pelo HSM para a fase de Rastreamento da Rede assenta em cinco passos em que o terceiro passo corresponde à seleção do(s) MIZAA(s). É especificamente neste passo que o presente estudo se centra. O HSM descreve 13 MIZAAs, cada um com caracteristicas especificas nomeadamente em termos de vantagens e limitações. No entanto, não é claro qual o MIZAA a selecionar para determinada aplicação e/ou estudo. O HSM aconselha a considerar sempre mais do que 1 método, sendo que a seleção deste depende de muitos fatores tais como, o objetivo e âmbito do trabalho, os dados disponíveis, a simplicidade e/ou precisão a considerar, etc. Na prática, o ranking dos locais por número de acidentes ou por taxa de acidentes são os MIZAAs mais comumente utilizados. Após a classificação dos locais através do MIZAA, a seleção dos locais a tratar baseia-se, em geral, num valor limite de número de acidentes ou numa condição de acordo com restrições de orçamento (Geedipally e Lord, 2010).

Mesmo depois de se analisar e decidir qual ou quais os MIZAA a aplicar é difícil garantir a precisão dos resultados. Com o objetivo de avaliar o desempenho dos vários MIZAAs, diversos autores têm vindo a desenvolver critérios de avaliação dos mesmos (Cheng e Washington, 2008, Montella, 2010, Cafiso e Silvestro, 2011, Lan e Persaud, 2011). Neste ponto é de notar que, tal como Cafiso e Silvestro (2011) sugerem, outras questões tais como o volume de tráfego, o comprimento das vias e o período de observação dos acidentes, podem enfatizar a qualidade dos resultados dos MIZAA. De facto, é difícil estabelecer uma clara definição de ZAA e, consequentemente uma correta identificação do local, pois uma simples observação de um número excecionalmente elevado de acidentes pode não significar necessariamente um problema de segurança relacionado com o local mas, pelo contrário, resultar de uma flutuação aleatória no período de observação considerado, habitualmente descrito como fenómeno de regresso à média (Elvik, 2008, Montella, 2010). Esta questão, entre outras, reflete-se numa errónea identificação de locais como ZAA resultando num número elevado de falsos negativos e falsos positivos tal como referido em Montella (2010). Locais identificados como falsos negativos são aqueles que são verdadeiramente perigosos mas foram identificados como seguros, e locais identificados como falsos positivos são aqueles que são verdadeiramente seguros mas foram identificados como perigosos.

Como se pode depreender, a identificação errada de locais como ZAA produz uma ineficiente alocação de recursos e consequentemente reduz a eficácia do processo de gestão de segurança implementado. Por este facto, alguns estudos têm-se centrado na avaliação do desempenho dos MIZAA considerando a análise dos falsos e verdadeiros positivos, e falsos e verdadeiros negativos sendo que a metodologia a considerar depende se a base de dados é empírica ou simulada (Geedipally e Lord, 2010, Cafiso e Silvestro, 2011, Lan e Persaud, 2011).

Mesmo optando pela aplicação de um MIZAA com bom desempenho, existe sempre um grau de incerteza associado à correta identificação do local como ZAA, principalmente se o método não considerar as características do local. Por exemplo, o método Bayes empírico baseia-se não só no número de acidentes observado, mas também no número estimado de acidentes com base na aplicação de um modelo de previsão de acidentes que incorpora as características do local.

Neste contexto, o presente estudo tem como objetivo principal constituir uma ferramenta de apoio à decisão na seleção e aplicação dos MIZAAs, contribuindo assim para a divulgação e aplicação de métodos de identificação de ZAA e seu posterior tratamento. Os 13 métodos propostos pelo HSM são apresentados de uma forma resumida e simples (em tabela). Os MIZAA são organizados segundo 3 critérios principais que, na perspetiva dos autores, pode- 
rão facilitar a consulta e posterior seleção do método a aplicar ao respetivo caso em estudo. Será ainda realizada uma aplicação e análise crítica dos métodos a um caso de estudo. Adicionalmente aos métodos propostos pelo HSM apresenta-se ainda neste estudo um método recentemente desenvolvido e designado de modelo binário probit (MBP) (Couto e Ferreira, 2013, Ferreira e Couto, 2013). Os resultados da aplicação deste elevado número de métodos ao caso da cidade do Porto foram comparados com base em testes de avaliação do desempenho tal como proposto por Cheng e Washington (2008) e Montella (2010).

\section{BREVE DESCRIÇÃO DO HSM - RASTREAMENTO DA REDE "NETWORK SCREENING"}

\subsection{Rastreamento da Rede "Network Screening”}

Nesta secção, pretende-se de uma forma resumida apresentar o capítulo 4, Rastreamento da Rede "Network Screening", do HSM. O referido capítulo propõe um procedimento de análise de uma rede viária com o objetivo de identificar e classificar os locais de acordo com o potencial do local em reduzir a frequência de acidentes através da implementação de medidas de tratamento. Este é o primeiro passo aconselhado pelo HSM para concretizar o processo de gestão de segurança rodoviária tal como descrito na parte $\mathrm{B}$ do mesmo manual. O procedimento proposto pelo HSM, para esta fase, assenta em cinco passos resumidos na Figura 1.

Como se pode constatar pela Figura 1, o primeiro passo consiste em estabelecer o foco da análise, ou seja, identificar o propósito e o objetivo da análise para examinar a rede viária. Segundo o HSM, o objetivo de uma análise deste tipo é:

- identificação e classificação de locais com potencial de redução do número de acidentes através de um conjunto de medidas;

- avaliação da rede para identificar locais onde ocorrem acidentes específicos, quer pelo tipo ou gravidade, ou ambos, de forma a formular e implementar políticas de segurança e prevenção.

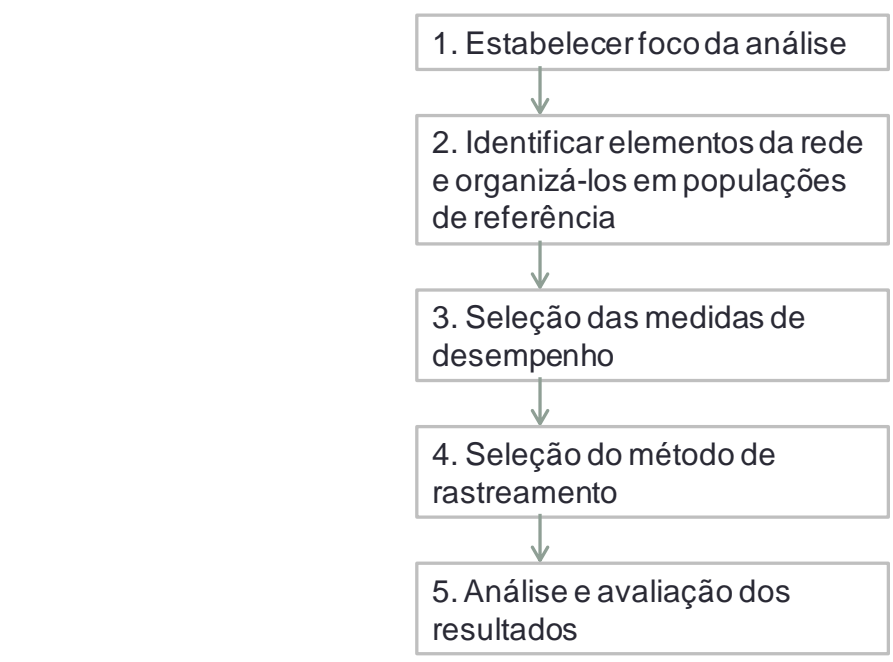

Figura 1 - Processo de Rastreamento da Rede "Network Screening Process" (Fonte:(AASHTO, 2010))

Esta decisão tem consequências no tipo de dados necessários para a análise, na seleção das medidas de avaliação de desempenho, bem como na seleção dos métodos a ser aplicados na análise.

$\mathrm{O}$ segundo passo resume-se a identificar os elementos da rede em estudo, e organiza-los segundo grupos, denominados populações de referência. Neste passo pretende-se especificar o tipo de locais a serem analisados (por exemplo, segmentos, interseções, cruzamentos de nível com via férrea) e também identificar grupos especificos desses mesmos locais (populações de referência). A população de referência é formada agrupando locais de características semelhantes (por exemplo, interseções de quatro ramos com sinalização luminosa). Neste caso, a seleção dos locais a tratar é realizada por população de referência. Assim, os MIZAA permitem uma análise dos locais dessa população de referência.

A definição das populações de referência 
depende do elemento escolhido para análise, ou seja, por exemplo, se a análise se centrar em interseções, as populações de referência podem ser estabelecidas segundo caraterísticas tais como o tipo de sinalização da interseção, o número de ramos, a classificação funcional das vias (arterial, coletora, local, etc), a área onde está inserida, intervalos de volumes de tráfego, etc. As caraterísticas que definem a população de referência podem variar dependendo da quantidade de detalhe conhecido e/ou possível de obter, do propósito da análise, da dimensão da rede a ser analisada, e mesmo do MIZAA selecionado. Neste último, se o MIZAA pretendido implicar, por exemplo, a aplicação de um modelo de previsão de acidentes, será necessário caracterizar a população de referência de acordo com as variáveis consideradas no modelo.

O terceiro passo corresponde à seleção dos MIZAAs. Existe uma grande variedade de MIZAA disponíveis para avaliar o potencial de um local para a redução da frequência de acidentes. Nesta etapa, o método é selecionado em função dos objetivos e critérios definidos nos passos anteriores dependendo, no entanto, dos dados e ferramentas analíticas disponíveis. Segundo o HSM, os principais critérios de seleção das medidas desempenho são a disponibilidade dos dados, o fenómeno regresso-à-média, e o modo como é definido o limite de desempenho. De facto, para uma análise profunda e fidedigna dos acidentes é necessário informação detalhada e precisa dos acidentes bem como a descrição das características geométricas dos locais onde estes ocorreram e os respetivos volumes de tráfego (tráfego médio diário anual, TMDA).

Relativamente aos fatores que contribuem para a qualidade da base de dados de acidentes a utilizar, o HSM refere essencialmente as limitações e dificuldades no registo dos dados, e na recolha e tratamento dos dados. Para ultrapassar as limitações de cada um dos MIZAA, o HSM propõe utilizar vários métodos em vez de um só método para avaliar os locais segundo perspetivas distintas aumentando desta forma o nível de confiança nos resultados.

No quarto passo é proposto a seleção do método de rastreamento para definir os locais prioritários a analisar. Neste ponto o HSM descreve três métodos principais (ranking, "sliding window", e "peak searching") bem como as vantagens e desvantagens de cada um. Os MIZAA devem ser aplicados a todos os locais em estudo utilizando o método, ou métodos de rastreamento que mais se adequem ao caso. De referir que os trés métodos de rastreamento são especificos ao caso de análise dos segmentos.

Por último, o passo cinco corresponde à análise e avaliação dos resultados. Este passo final consiste em avaliar os resultados obtidos tendo em conta os objetivos definidos no primeiro passo, sugerindo-se a organização dos resultados num quadro ou num mapa.

\subsection{Método de Identificação de Zonas de Acumulação de Acidentes}

Na Tabela 1 resume-se os 13 MIZAA propostos pelo HSM tendo em conta os dados necessários, vantagens e limitações de cada método. Na última coluna, está indicado os métodos que foram considerados ou não na aplicação ao caso de estudo. Nesta tabela está também incluído o MBP tal como proposto por (Couto e Ferreira, 2013, Ferreira e Couto, 2013). Tendo como base as conclusões de um estudo no âmbito de uma tese de mestrado (Martins, 2013) que envolveu uma análise aprofundada e a aplicação dos MIZAA propostos pelo HSM, apresenta-se neste artigo os métodos organizados com base em 3 critérios de aplicação: dados de volume tráfego (com ou sem TMDA), função de previsão de acidentes (com ou sem FPA) e população de referência (com ou sem separação de populações de referência).

A opção de diferenciar os MIZAA segundo estes critérios baseia-se no facto de ser muito comum não existir ou não ser possível obter dados fiáveis do TMDA em algumas cidades, regiões ou países. Este facto excluiu logo à partida a seleção de determinados métodos. É o caso também dos métodos que se baseia na aplicação de uma FPA desenvolvida ou calibrada para as condições locais, para estimar a frequência média de acidentes para cada local. Neste caso é necessário não só ter dados de acidentes e de TMDA como também dispor de conhecimentos, meios e tempo para desenvolver com um nível de confiança estatístico elevado uma FPA. Embora, tal como o HSM refere, idealmente uma FPA deveria ser desenvolvida para as condições locais com uma base de dados da área em estudo, o HSM oferece uma alternativa a desenvolver as próprias FPA. Uma 
grande parte do manual (parte $\mathrm{C}$ e parte $\mathrm{D}$ ) é dedicada à descrição de uma metodologia de aplicação de FPA em função do tipo de entidade viária (interseção ou segmento), e ao ajuste do valor estimado às caracteristicas específicas do local através dos fatores de modificação de sinistralidade (FMSs). O último passo desta metodologia corresponde à calibração dos resultados obtidos pela aplicação da metodologia do HSM ao caso em estudo. Este passo tem sido o foco de diversos estudos (Persaud, et al., 2002, Sawalha e Sayed, 2006, Chen, et al., 2012, Sacchi, et al., 2012).

Há ainda a diferenciar os MIZAA do HSM que são aplicados separadamente por população de referência. Neste caso, para além de ser necessário definir populações de referência, a própria aplicação e respetivos resultados são separados, condicionando, por isso, a comparação conjunta dessas populações. Nestes métodos cada local é avaliado relativamente ao conjunto dos locais da mesma população. Notese que apesar de alguns métodos referirem como necessário definir populações de referência, não estão, no entanto, condicionados à separação dos resultados e respetiva agregação. Nestes casos, a(s) populações de referência são necessários para aplicar os procedimentos do método.

A Tabela 1 resume e organiza os MIZZA propostos pelo HSM bem como o MBP. Este último método baseia-se num modelo probabilístico cuja variável de resposta corresponde à definição de um local como ZAA ou não-ZAA. A probabilidade de um local ser ou não ZAA é determinada em função das características principais desse mesmo local. Por outro lado, a definição de ZAA baseia-se na utilização de um valor limite para o número de acidentes acima do qual se considera o local como ZAA (Couto e Ferreira, 2013).

Tabela 1 - Descrição resumida dos MIZAAs

(continua)

\begin{tabular}{|c|c|c|c|c|c|}
\hline $\begin{array}{l}\text { Critérios de } \\
\text { aplicação }\end{array}$ & MIZAA & Dados necessários & Vantagens & Limitações & $\begin{array}{l}\text { Aplicação ao } \\
\text { caso de es- } \\
\text { tudo }\end{array}$ \\
\hline $\begin{array}{l}\text { Sem TMDA } \\
\text { Sem FPA }\end{array}$ & $\begin{array}{l}\text { FMA - Fre- } \\
\text { quência Mé- } \\
\text { dia de Aci- } \\
\text { dentes }\end{array}$ & $\begin{array}{l}\text { - Dados de acidentes por } \\
\text { localização }\end{array}$ & - Simples; & $\begin{array}{l}\text { - Não considera o fenóme- } \\
\text { no de regresso-à-média; } \\
\text { - Não estima um limite que } \\
\text { permite indicar locais onde } \\
\text { ocorrem mais acidentes do } \\
\text { que o previsto para locais } \\
\text { de características seme- } \\
\text { Ihantes; } \\
\text { - Não considera o volume } \\
\text { de tráfego; } \\
\text {-Não identifica locais com } \\
\text { baixo número de colisões, } \\
\text { nas quais medidas simples } \\
\text { de custo -benefício poderi- } \\
\text { am ser facilmente aplica- } \\
\text { das. }\end{array}$ & $\begin{array}{l}\text { Sim (ao nú- } \\
\text { mero total de } \\
\text { acidentes) }\end{array}$ \\
\hline $\begin{array}{l}\text { Sem TMDA } \\
\text { Sem FPA }\end{array}$ & $\begin{array}{l}\text { EADM - Fre- } \\
\text { quência mé- } \\
\text { dia de aci- } \\
\text { dentes equi- } \\
\text { valente ape- } \\
\text { nas a danos } \\
\text { materiais }\end{array}$ & $\begin{array}{l}\text { - Dados de acidentes por } \\
\text { localização; } \\
\text { - Fatores de ponderação } \\
\text { de gravidade (vítima } \\
\text { mortal, feridos, danos } \\
\text { materiais); } \\
\text { - Custo de acidente por } \\
\text { gravidade (vítima mortal, } \\
\text { feridos, danos materiais). }\end{array}$ & $\begin{array}{l}\text { - Simples; } \\
\text { - Considera a } \\
\text { gravidade do } \\
\text { acidente. }\end{array}$ & $\begin{array}{l}\text { - Não considera o fenóme- } \\
\text { no de regresso-à-média; } \\
\text { - Não estima um limite que } \\
\text { permite indicar locais onde } \\
\text { ocorrem mais acidentes do } \\
\text { que o previsto para locais } \\
\text { de características seme- } \\
\text { lhantes; } \\
\text { - Não considera volume de } \\
\text { tráfego; } \\
\text { - Pode sobrevalorizar lo- } \\
\text { cais com frequência baixa } \\
\text { de acidentes graves de- } \\
\text { pendendo dos fatores de } \\
\text { ponderação utilizados. }\end{array}$ & $\begin{array}{l}\text { Não, por falta } \\
\text { de dados } \\
\text { relativos aos } \\
\text { custos }\end{array}$ \\
\hline
\end{tabular}




\begin{tabular}{|c|c|c|}
\hline $\begin{array}{l}\text { Critérios de } \\
\text { aplicação }\end{array}$ & MIZAA & Dados necessários \\
\hline $\begin{array}{l}\text { Sem TMDA } \\
\text { Sem FPA }\end{array}$ & $\begin{array}{l}\text { IGR - Índice } \\
\text { de gravidade } \\
\text { relativa }\end{array}$ & $\begin{array}{l}\text { - Dados de acidentes por } \\
\text { localização; } \\
\text { - Custos de acidente IGR } \\
\text { (custo de acidente de } \\
\text { colisão lateral em inter- } \\
\text { seção com sinais lumino- } \\
\text { sos, custo de acidente de } \\
\text { colisão lateral em inter- } \\
\text { seção de prioridade à } \\
\text { direita, custo de acidente } \\
\text { de colisão lateral em } \\
\text { interseção prioritária, e } \\
\text { restantes custos relativos } \\
\text { a outros tipos de aciden- } \\
\text { te); } \\
\text { - População de referên- } \\
\text { cia. }\end{array}$ \\
\hline
\end{tabular}

EMM - Ex-

cesso previsto da frequência média de acidentes Método dos Momentos
- Dados de acidentes por localização;

- População de referência.
- Simples.

- Considera o

tipo de aciden-

te e gravidade.
Sem TMDA Sem FPA

\begin{tabular}{lll} 
Vantagens $\quad$ Limitações & $\begin{array}{l}\text { Aplicação ao } \\
\text { caso de es- } \\
\text { tudo }\end{array}$ \\
\hline
\end{tabular}

- Não considera o fenómeno de regresso-à-média; - Pode enfatizar locais com um baixo número de acidentes graves dependendo do fator de ponderação utilizado;

- Não considera volume de tráfego;

- Pode erradamente dar prioridade a locais de potencial baixo volume de tráfego, e baixo número de acidentes
Não, por falta de dados relativos aos custos
- Estabelece um limite de desempenho previsto para um local;

- Considera a variância dos dados de acidentes;

- Permite posicionar numa única lista os locais de todos os tipos;

- Os conceitos metódicos do método são semelhantes ao método Empírico de

Bayes.
PEPL - Probabilidade de tipos específicos de acidentes excederem uma percentagem limite
- Dados de acidentes por tipo e localização; - População de referência.
- Os efeitos do fenómeno regresso-à-média podem estar presentes nos resultados;

- Não considera o volume de tráfego;

- Alguns locais podem ser selecionados para um estudo mais profundo, devido à incomum frequência baixa de tipos de acidentes não considerados;

- A classificação dos resultados é influenciada pela população de referência; os locais perto da fronteira do grupo de referência podem ser enfatizados
- Pode ser utilizado como ferramenta de diagnóstico;

- Considera a variância dos dados de acidentes: - Não é afetado pelo fenómeno de regresso-àmédia.
- Não contabiliza o volume de tráfego;

- Alguns locais podem ser selecionados, para serem aprofundados mais tarde devido à incomum frequência baixa de tipos de acidentes não objetivados.
Não por ser aplicado a tipos específicos de acidentes e como tal sujeito a erros de classificação do tipo de acidente; não comparável com a aplicação dos outros métodos Não por ser aplicado a tipos específiutilizado como ferramenta de diagnóstico;

- Considera a variância dos dados de acidentes; - Não é afetado pelo fenómeno de regresso-àmédia.
- Não contabiliza o volume de tráfego;

- Alguns locais podem ser selecionados, para serem aprofundados mais tarde devido à incomum frequência baixa de tipos de acidentes não objetivados. cos de aci-

dentes e como tal sujeito a erros de classificação do tipo de acidente; não comparável com a aplicação dos outros métodos 


\begin{tabular}{lll}
\hline $\begin{array}{l}\text { Critérios de } \\
\text { aplicação }\end{array}$ & MIZAA & Dados necessários \\
\hline & & \\
& & \\
$\begin{array}{ll}\text { Com TMDA } \\
\text { Sem FPA }\end{array}$ & $\begin{array}{l}\text { TA - Taxa de } \\
\text { acidentes }\end{array}$ & $\begin{array}{l}\text { D Dados de acidentes por } \\
\text { localização; } \\
\text { • Volume de tráfego. }\end{array}$
\end{tabular}

\begin{tabular}{|c|c|}
\hline Vantagens & Limitações \\
\hline
\end{tabular}

- Simples;

- Se a organização dos dados de acidentes for a mesma que foi utilizada nos métodos EP. DO e IGR, o método taxa de acidentes pode ser modificado para considerar a gravidade.
- Não considera o fenómeno regresso-à-média; - Não estima um limite que permite indicar locais onde ocorrem mais acidentes do que o previsto em comparação com locais de características semelhantes; - Não podem ser feitas comparações entre locais com diferenças significativas de volume de tráfego; - Poderá erradamente dar prioridade a locais de baixo volume de tráfego, locais come baixo número de colisões

- Reduz o

efeito exagerado de locais com baixo volume;

- Dados de acidentes por

TCA - Taxa localização;
- Não considera o fenómeno regresso-à-média;

Sim crítica de

- Volume de tráfego; acidentes

- Populações de referência.

- Considera a variância dos dados de acidentes; - Estabelece um limite para comparação.

- Dados de acidentes por localização (recomendado um período de 3 a 5 anos);
Com TMDA

Com FPA

Aplicado a

População de

Referência

NSS - Nível de serviço de segurança - Função de previsão de acidentes (FPA) calibrada e parâmetro de sobredispersão;

- Volume de tráfego (no caso das interseções separado em principal e secundário);

- População de referência.

- Dados de acidentes por localização;

EPFA - Ex-

Com TMDA Com FPA

Aplicado a População de Referência cesso previsto da frequência média de acidentes FPAs
- População de referência;

- Volume de tráfego (no caso das interseções separado em principal e secundário);

- Função de previsão de acidentes (FPA) calibrada e parâmetro de sobredispersão.

\section{- Considera a} variância dos dados de acidentes;

- Considera o - Os efeitos do fenómeno volume de tráfego;

- Estabelece um limite para medir a frequência de acidentes. de regresso-à-média podem estar presentes nos resultados.

\section{Sim}

Sim, apenas às interseções com sinais luminosos.
- Considera o volume de tráfego;

- Estabelece um limite para comparação.
- Os efeitos do fenómeno regresso-à-média podem estar presentes nos resultados.
Sim, apenas às interseções com sinais luminosos. 


\begin{tabular}{|c|c|c|c|c|c|}
\hline $\begin{array}{l}\text { Critérios de } \\
\text { aplicação }\end{array}$ & MIZAA & Dados necessários & Vantagens & Limitações & $\begin{array}{l}\text { Aplicação ao } \\
\text { caso de es- } \\
\text { tudo }\end{array}$ \\
\hline
\end{tabular}

$\begin{array}{ll}\text { Com TMDA } & \text { MEB - } \\ \text { Com FPA } & \text { Frequência } \\ \text { Aplicado a } & \text { média de } \\ \text { População de } & \text { acidentes } \\ \text { Referência } & \text { esperada - } \\ & \text { Método Em- } \\ & \text { pírico de } \\ & \text { Bayes }\end{array}$

Com FPA

Aplicado a

População de

Referência

EADM MEB-

Frequência média de acidentes equivalente apenas a danos mate-
- Dados de acidentes por localização;

- População de referência;

- Volume de tráfego (no caso das interseções separado em principal e secundário);

- Características básicas das interseções (por exemplo, tipo de sinalização, número de ramos);

- FPA calibrada e parâmetro de sobredispersão.

- Dados de acidentes por gravidade e localização;

- Fatores de ponderação riais (EADM) - Método Empírico de Bayes (MEB) exemplo, tipcoes (por zação, número de ramos);
- Considera o fenómeno regresso-àmédia.
- Necessita da FPA calibrada para as condições locais.

Sim, apenas às interseções com sinais luminosos.

\section{- Considera o}

fenómeno regresso-àmédia;

- Considera a gravidade do acidente.
- Pode sobrevalorizar locais de baixo número de acidentes graves, dependendo dos fatores de ponderação utilizados.
Não por ser aplicado a acidentes por gravidade e como tal sujeito a erros de classificação; não comparável com a aplicação dos outros métodos

- FPA calibrada e parâmetro de sobredispersão.

- Dados de acidentes por localização;

- População de referên-

EMEB - Excesso espe-

Com TMDA Com FPA População de Referência rado da frequência média de acidentes Método Empírico de Bayes
Aplicado a

cia;

- Volume de tráfego (no

caso das interseções separado em principal e secundário);

- Características básicas das interseções (por exemplo, tipo de sinalização, número de ramos);

- FPA calibrada e parâmetro de sobredispersão.

- Dados de acidentes por localização;

- Volume de tráfego (no caso das interseções

Com TMDA

Com modelo probabilístico
MBP - Mode-

lo binário probit separado em principal e secundário);

- Eventualmente características básicas das interseções (por exemplo, tipo de sinalização, número de ramos);

- Modelo binário.

\section{- Considera o}

fenómeno

regresso-à-

média;

- Identifica um

valor limite

que permite

indicar locais

onde ocorrem

- Nenhuma.

Sim, apenas às interseções mais acidentes do que o especom sinais luminosos rado, compa-

rativamente a

locais de ca-

racterísticas semelhantes.

\section{- Considera o}

fenómeno

regresso-à-

média;

- Considera o

volume de

tráfego;

- Determina a

- Necessita de um modelo

probabilidade

binário calibrado para as

condições locais.
Sim

do local ser

ZAA; 


\section{APLICAÇÃO AO CASO DE ESTUDO - INTERSEÇÕEES DA CIDADE DO PORTO}

Nesta secção descreve-se a seguir em 3.1., a base de dados da cidade do Porto, Portugal relativa aos anos 2001 a 2005. Esta base de dados será utilizada para a aplicação dos diferentes MIZAA. Como se depreende pela Tabela 1, alguns MIZAA necessitam de uma FPA e o último método (MBP) necessita de um modelo binário. Estes modelos foram desenvolvidos para as condições locais. Na secção 3.2 são descritos estes modelos estatísticos.

\subsection{Descrição da base de dados}

Os dados utilizados neste estudo são relativos a acidentes ocorridos em interseções da cidade do Porto de 3 e 4 ramos, e com ou sem sinalização luminosa, registados ao longo de um período de 5 anos (de 1 de Janeiro de 2001 a 31 de Dezembro de 2005). A base de dados dos acidentes foi obtida a partir de dados ofici- ais da Polícia de Segurança Pública e incluem todo o tipo de acidentes (com vítimas e só com danos materiais) registados com a informação do local de ocorrência. Com base nesta informação, os acidentes foram georreferenciados através de um sistema de informação geográfica. Os dados consistem em 2029 acidentes, dos quais 447 resultaram em vítimas e 1582 só com danos materiais, referenciados a 211 interseções. Estas interseções estão divididas em 48 interseções de 3 ramos e sinalização luminosa; 67 interseções de 3 ramos sem sinalização luminosa; 70 interseções de 4 ramos e sinalização luminosa; e 26 interseções de 4 ramos e sem sinalização luminosa. A Tabela 2 apresenta a descrição estatística relativa aos acidentes e ao TMDA das interseções da cidade do Porto.

Tabela 2 - Descrição estatística da base de dados do Porto

\begin{tabular}{lllll}
\hline Variável & Min. & Máx. & Média & Desvio Padrão \\
\hline Número de acidentes & 0 & 13 & 1,9 & 2,1 \\
TMDA & 285 & 71525 & 18309 & 11629 \\
TMDA & 0 & 32882 & 5286 & 5232 \\
\hline
\end{tabular}

\subsection{Aplicação dos MIZAA}

Para os MIZAA que necessitam de uma FPA optou-se por desenvolver uma função para as condições locais a partir da qual se estimaram os parâmetros das variáveis explanatórias para uma regressão cuja estrutura do erro assume uma distribuição binomial negativa $(\mathrm{BN})$ tal como proposto pelo HSM. Estes valores estão apresentados na Tabela 3. A distribuição $\mathrm{BN}$ permite representar a sobre-dispersão geralmente verificada nas bases de dados de acidentes entre locais através do parâmetro de dispersão (AASHTO, 2010).

\begin{tabular}{cccc}
\multicolumn{4}{c}{ Tabela 3 - Resultados do modelo BN para a base de dados do Porto } \\
\hline Parâmetro & Valor estimado & Desvio padrão & P[Z>Z $]$ \\
\hline Constante & $-3,175$ & 0,467 & 0,0000 \\
Ln(TMDAPrinc) & 0,303 & 0,049 & 0,0000 \\
Ln(TMDAsec) & 0,076 & 0,011 & 0,0000 \\
4Ramos & 0,126 & 0,067 & 0,0606 \\
Sinal. Luminosa & 0,409 & 0,066 & 0,0000 \\
Parâmetro dispersão & 0,502 & 0,052 & 0,0000 \\
\hline
\end{tabular}

Relativamente ao modelo binário este foi aplicado à base de dados considerando para as categorias $(0 / 1)$ da variável dependente, que distinguem o local como ZAA (categoria 1) ou não ZAA (categoria 0), um valor fronteira. Após uma análise de várias hipóteses, considerou-se o percentil 80 e que corresponde a 3 acidentes por intersecção em média no período de 5 anos em estudo. Assim, aos locais com um número de acidentes igual ou superior a 3 , foi atribuída a categoria 1 e classificados como ZAA, e aos restantes locais a categoria 0 (para mais desenvolvimentos do MBP ver o artigo de Couto e Ferreira (2013)). 


\begin{tabular}{cccc}
\multicolumn{4}{c}{ Tabela 4 - Resultados do modelo binário probit para a base de dados do Porto } \\
\hline Parâmetro & $\begin{array}{c}\text { Valor } \\
\text { estimado }\end{array}$ & Desvio Padrão & P[Z>Z $]$ \\
\hline Constante & $-4,568$ & 0,695 & 0,0000 \\
Ln(TMDA $\left.A_{\text {Princ }}\right)$ & 0,312 & 0,071 & 0,0000 \\
Ln(TMDA & 0,092 & 0,016 & 0,0000 \\
4Ramos & 0,141 & 0,090 & 0,1178 \\
Sinal. Luminosa & 0,434 & 0,099 & 0,0000 \\
\hline
\end{tabular}

Considerando a distribuição probit, obtiveram-se os resultados para o MBP apresentados na Tabela 4. Como se pode verificar, os parâmetros das variáveis explanatórias foram estimados com um nível de confiança de $95 \%$, exceto a variável correspondente ao número de ramos da interseção. Contudo, optou-se por manter o valor estimado no cálculo do método por ser um valor próximo do nível de confiança de $90 \%$ bem como do nível de confiança do valor estimado pelo modelo BN (ver Tabela 3 ). Além disso, os parâmetros estimados apresentam valores coerentes com as expectativas.

\section{COMPARAÇÃO DOS MIZAA PARA O CASO DE ESTUUDO}

Nesta secção descreve-se a seguir em 4.1., a metodologia utilizada para a comparação dos MIZAA aplicados ao caso da cidade do Porto. Posteriormente, na secção 4.2 são mostrados os números de identificação (ID) das intersecções selecionadas segundo os vários métodos aplicados. Neste caso, dividiu-se os resultados em duas tabelas considerando que alguns dos métodos foram aplicados a todas as intersecções e outros métodos foram aplicados a uma população de referência - intersecções com sinalização luminosa.

\subsection{Metodologia}

Como os dados considerados neste estudo são empíricos, a escolha da metodologia a utilizar para a avaliação do desempenho dos MIZAA é condicionada a este facto, pois não é possível aferir e comparar com os "verdadeiros" locais ZAA. Assim, e à semelhança de estudos anteriores (Cheng e Washington, 2008, Montella, 2010, Ferreira e Couto, 2012), o período de 5 anos em estudo foi dividido em dois: 2001 a 2002 (2 anos) e 2003 a 2005 (3 anos) de forma a ser possível comparar a consistência dos resultados da aplicação dos MIZAA nos dois períodos e assim avaliar o desempenho. Para estes dois períodos de tempo foram apli- cados 4 testes que permitem avaliar o desempenho dos MIZAA aplicados ao caso de estudo.

$O$ teste 1 é designado de consistência do local e tem como objetivo comparar o número total de acidentes ocorridos no primeiro período com o segundo período para os locais selecionados como ZAA no primeiro período, tal como descreve a equação abaixo:

Teste 1= $\sum_{\mathrm{k}=1}^{\mathrm{n}} \mathrm{C}_{\mathrm{k}, \mathrm{m}=\mathrm{z} ; \text { Periodo } 2}>$
$\sum_{\mathrm{k}=1}^{\mathrm{n}} \mathrm{C}_{\mathrm{k}, \mathrm{m}=\mathrm{v} \neq \mathrm{z} ; \text { Periodo2 }}$

Em que $C$ representa o número de acidentes para o local $k, N$ corresponde ao total de locais e $v, z$ os métodos $m$ em comparação. Neste teste, quanto maior o numero de acidentes total obtido melhor o desempenho do método.

O teste 2 avalia a consistência do método, ou seja, compara o número de locais selecionados como ZAA no primeiro período que continuaram a ser selecionados como ZAA, no segundo período. Analiticamente, o teste 2 é obtido pela interseção dos locais identificados como ZAA nos dois períodos em análise. Assim, o método com melhor desempenho é aquele com o maior número de locais em comum aos dois períodos.

O teste 3 designado de diferença total do ranking corresponde a comparar, entre os dois períodos temporais, o ranking dos locais selecionados no primeiro período como ZAA calculando o total da diferença absoluta entre a posição no ranking do primeiro com a posição no ranking do segundo período. A equação abaixo descreve o teste 3 :

Teste $3=\sum_{\mathrm{k}=1}^{\mathrm{n}}\left|\mathrm{R}\left(\mathrm{k}_{\mathrm{m}, \text { Periodo1 }}\right)-\mathrm{R}\left(\mathrm{k}_{\mathrm{m}, \text { Periodo2 }}\right)\right|$

Em que $R$ é a posição no ranking do local $k$ no Período 1 e no Período 2 para o método $m$. 
Neste caso, quanto menor o valor da diferença melhor.

Estes três testes foram desenvolvidos por Cheng e Washington (2008). Posteriormente e na sequência dos testes anteriores, Montella (2010) desenvolveu um teste designado de pontuação total que pontua de 0 a 100 os MIZAA combinando os resultados dos três testes anteriores. $\mathrm{O}$ teste pontuação total atribui o mesmo peso para cada um dos três testes. Quanto maior o valor deste teste melhor o desempenho do método e se atingir a pontuação 100 significa que o método é o melhor em todos os três testes.

\subsection{Seleção dos locais segundo os diferentes MIZAA}

Com o objetivo de comparar os diferentes MIZAA considerou-se o mesmo número de interseções selecionadas como sendo ZAA por cada método, tendo-se optado por $10 \%$ do total da amostra a estudar. No caso dos métodos aplicados a uma população de referência, considerou-se a aplicação dos respetivos métodos ao caso das interseções com sinais luminosos. Tendo em conta o número elevado de métodos e locais bem como os diferentes procedimentos dos métodos que por vezes não correspondem a um valor numérico final, optou-se por apresen- tar para cada MIZAA, como resultado da aplicação dos vários métodos, o ID das interseções selecionados como ZAA por cada método no período de referência 2001-2002. Assim, a Tabela 5 apresenta as 21 ZAAs selecionadas pelos vários métodos aplicados a todo o tipo de interseções (211) e na Tabela 6 são apresentados os ID das interseções com sinais luminosos selecionadas como ZAA (12 interseções) pelos respetivos métodos. A ordenação dos ID corresponde à ordem decrescente de gravidade do local como ZAA segundo o método aplicado.

Da Tabela 5 é possível concluir que não há nenhuma interseção (ID) comum a todos os 5 métodos aplicados. Tendo como referência o método FMA, pode-se referir que os métodos FMA e EEM selecionaram o maior número de interseções comuns (isto é, com o mesmo ID), num total de 17 interseções comuns em 21 das interseções selecionada por cada método. $\mathrm{O}$ método MBP relativamente ao método FMA tem apenas 6 ID comuns e nenhum ID em comum com o TA, por exemplo. Pelo contrário, e tal como seria expectável, nos métodos TA e TCA que se baseiam numa taxa de acidentes, apenas 2 interseções não são comum aos dois métodos e a posição na ordem do ranking é também muito próxima entre os métodos.

Tabela 5 - Número de identificação (ID) das interseções selecionadas como ZAA pelos diferentes MIZAA

\begin{tabular}{|c|c|c|c|c|}
\hline \multicolumn{5}{|c|}{$\begin{array}{c}\text { ID Interseções } \\
2001-2002\end{array}$} \\
\hline $\begin{array}{l}\text { FMA } \\
\text { Frequência Média de } \\
\text { Acidentes }\end{array}$ & $\begin{array}{l}\text { EMM } \\
\text { Excesso previsto da frequência média de } \\
\text { acidentes - Método dos Momentos }\end{array}$ & $\begin{array}{l}\text { TA } \\
\text { Taxa de Aci- } \\
\text { dentes }\end{array}$ & $\begin{array}{l}\text { TCA } \\
\text { Taxa Crítica de } \\
\text { Acidentes }\end{array}$ & $\begin{array}{l}\text { MBP } \\
\text { Modelo Biná- } \\
\text { rio Probit }\end{array}$ \\
\hline 147 & 128 & 93 & 122 & 150 \\
\hline 128 & 124 & 122 & 99 & 211 \\
\hline 115 & 194 & 99 & 165 & 201 \\
\hline 99 & 193 & 165 & 114 & 7 \\
\hline 114 & 147 & 114 & 192 & 205 \\
\hline 205 & 99 & 189 & 154 & 42 \\
\hline 15 & 114 & 151 & 124 & 144 \\
\hline 187 & 115 & 192 & 194 & 184 \\
\hline 142 & 50 & 154 & 193 & 182 \\
\hline 124 & 15 & 90 & 115 & 31 \\
\hline 194 & 142 & 1 & 151 & 46 \\
\hline 46 & 187 & 124 & 93 & 15 \\
\hline 193 & 205 & 115 & 1 & 187 \\
\hline 7 & 11 & 193 & 90 & 69 \\
\hline 52 & 46 & 194 & 189 & 51 \\
\hline 127 & 7 & 110 & 39 & 198 \\
\hline 150 & 52 & 39 & 112 & 29 \\
\hline 25 & 127 & 112 & 119 & 6 \\
\hline 146 & 85 & 156 & 11 & 16 \\
\hline 14 & 179 & 185 & 110 & 30 \\
\hline 119 & 14 & 119 & 14 & 77 \\
\hline
\end{tabular}


Da Tabela 6 pode-se concluir que as interseções com ID 147, 99, 115 e 5 são comuns aos 5 métodos aplicados em que, por exemplo, o ID 147 está posicionado na lista de ranking em primeiro lugar em 3 dos 5 métodos. Considerando como base de comparação o método FMA, concluiu-se ainda que através dos métodos EPFA e EMEB selecionaram-se 10 e 11 interseções num total de 12 em comum, respetivamente com o FMA.
Para melhor avaliar o desempenho de cada método considerou-se a aplicação dos quatro testes de avaliação do desempenho a dados empíricos tais como propostos por Cheng e Washington (2008) e Montella (2010) e descritos anteriormente. Assim, na Tabela 7 são apresentados os resultados dos quatro testes para cada MIZZA nas duas situações em análise (com e sem população de referência).

Tabela 6 - Número de identificação (ID) das interseções com sinais luminosos (população de referência) selecionadas como ZAA pelos diferentes MIZAA

ID Interseções
$2001-2002$

\begin{tabular}{|c|c|c|c|c|}
\hline $\begin{array}{l}\text { FMA } \\
\text { Frequência } \\
\text { Média de Aci- } \\
\text { dentes }\end{array}$ & $\begin{array}{l}\text { NSS } \\
\text { Nível de serviço } \\
\text { de segurança }\end{array}$ & $\begin{array}{l}\text { EPFA } \\
\text { Excesso previs- } \\
\text { to da frequên- } \\
\text { cia média de } \\
\text { acidentes - } \\
\text { FPAs }\end{array}$ & $\begin{array}{l}\text { MEB } \\
\text { Frequência } \\
\text { média de aci- } \\
\text { dentes espera- } \\
\text { da - Método } \\
\text { Empírico de } \\
\text { Bayes }\end{array}$ & $\begin{array}{l}\text { EMEB } \\
\text { Excesso espe- } \\
\text { rado da fre- } \\
\text { quência média } \\
\text { de acidentes - } \\
\text { Método Empíri- } \\
\text { co de Bayes }\end{array}$ \\
\hline 147 & 114 & 147 & 114 & 147 \\
\hline 99 & 115 & 187 & 115 & 99 \\
\hline 114 & 99 & 205 & 99 & 115 \\
\hline 115 & 122 & 142 & 147 & 15 \\
\hline 15 & 127 & 15 & 15 & 142 \\
\hline 142 & 119 & 46 & 127 & 187 \\
\hline 187 & 147 & 7 & 142 & 114 \\
\hline 205 & 14 & 99 & 119 & 205 \\
\hline 46 & 117 & 150 & 14 & 127 \\
\hline 7 & 84 & 52 & 187 & 46 \\
\hline 52 & 15 & 25 & 122 & 14 \\
\hline 127 & 154 & 115 & 117 & 52 \\
\hline
\end{tabular}

Tabela 7 - Resultados dos testes de avaliação do desempenho dos MIZAA

\begin{tabular}{cccccccccccc}
\hline & \multicolumn{3}{c}{ Total das Interseções } & \multicolumn{3}{c}{$\begin{array}{l}\text { Interseções com sinais luminosos } \\
\text { (população de referência) }\end{array}$} \\
\hline & FMA & EMM & TA & TCA & MBP & FMA & NSS & EPFA & MEB & EMEB \\
\hline Teste 1(n- total de acidentes) & $\mathbf{8 4}$ & 77,7 & 54 & 57 & 58,7 & 50,3 & 38,7 & $\mathbf{5 6}$ & 44,3 & 46,3 \\
Teste 2 (\% de locais comuns) & 47,6 & 42,9 & 42,9 & 42,9 & $\mathbf{8 5 , 7}$ & 41,7 & 25,0 & $\mathbf{5 0 , 0}$ & $\mathbf{5 0 , 0}$ & 41,7 \\
Teste 3 (diferença ranking) & 676 & 792 & 1156 & 1343 & $\mathbf{7 0}$ & 256 & 463 & $\mathbf{1 5 2}$ & 281 & 303 \\
Teste Pontuação Total & 70,1 & 62,9 & 44,5 & 41,0 & $\mathbf{8 9 , 9}$ & 83,0 & $\mathbf{4 8 , 7}$ & $\mathbf{1 0 0}$ & 83,0 & $\mathbf{7 6 , 9}$ \\
\hline
\end{tabular}

$\mathrm{Na}$ Tabela 7 identificou-se a negrito os melhores resultados de cada teste. Como se pode verificar pela Tabela 7, no caso da comparação dos MIZAA quando aplicados a todas as interseções, o método com melhor desempenho é o MBP. Apenas no teste 1 este método não é o melhor indicando que os locais selecionados não são consistentes por não apresentarem um número total de ocorrências elevado no segundo período temporal. É, no entanto, o método mais consistente na seleção dos mesmos locais como ZAA (teste 2) e mais consistente no posicionamento no ranking da lista de ZAAs (teste 3). Segundo o teste 1, o melhor método é o FMA. O método com pior desempenho segun- do o teste pontuação total é o TCA. No caso da comparação dos MIZAA aplicados à população de referência, é evidente que o método com melhor desempenho em qualquer dos testes é o EPFA pois a pontuação total é de 100 e o método com menor pontuação total é o NSS.

\section{CONSIDERAÇÕES FINAIS}

O HSM é um importante manual que potencializa a utilização de metodologias por parte dos profissionais da segurança viária, contribuindo assim para a diminuição da sinistralidade. No entanto, dada a vasta informação contida neste manual nem sempre é fácil de uma 
forma simples e rápida tomar decisões nomeadamente na seleção dos métodos de identificação de zonas de acumulação de acidentes (MIZAA). Este ponto é pois o foco deste estudo. De facto, o HSM propõe um número elevado de métodos com características muito distintas. Acresce o facto de que estes métodos poderão ter comportamentos distintos conforme o contexto viário em que são aplicados, sendo por isso importante aplicar e avaliar estes métodos em diferentes contextos.

Com o presente estudo foi possível aferir as vantagens e limitações de cada método ao contexto de uma cidade portuguesa. Foram assim identificados métodos que não são possíveis, até à data, de aplicar em Portugal de uma forma fiável por falta de dados/informação, como é exemplo, os custos dos acidentes. Para além dos MIZAA propostos pelo HSM foi ainda aplicado o modelo binário probit (MBP) recentemente desenvolvido por Couto e Ferreira (2013) e cujos resultados obtidos neste estudo estão de acordo com os resultados dos estudos anteriores na medida em que se revelou como o método com melhor desempenho segundo a metodologia de comparação utilizada. De facto, segundo 3 dos 4 testes de avaliação de desempenho, o método MBP revelou ser o melhor. Observando os ID das interseções selecionadas por cada método, pode-se concluir que o método MBP selecionou interseções distintas das dos outros métodos, o que salienta o facto de este método se distinguir dos restantes. De notar, no entanto que estes resultados são referentes ao estudo de todas as interseções. $\mathrm{Na}$ aplicação e análise dos métodos aplicados a uma população de referência, no caso interseções com sinais luminosos, o método que se revelou mais eficiente segundo os quatro testes foi o excesso esperado da frequência média de acidentes - método empírico de Bayes (EMEB).

Por não ser possível estender o presente estudo, não se descreve aqui a análise crítica de cada método, mas que foi realizada e pode ser consultada no documento (Martins, 2013). Como se referiu acima, e na opinião dos autores, o desenvolvimento deste tipo de estudos será claramente enriquecido se aplicado nos mesmos moldes a outros contextos viários.

\section{REFERÊNCIAS}

AASHTO (2010) Highway Safety Manual. American Association of State Highway and Transportation Officials, Washington DC. ISBN: 978-1-56051-477-0

Bandyopadhyaya, R. e Mitra, S. (2011) Comparative Analysis of Hotspot Identification Methods in the Presence of Limited Information. 3rd International Conference on Road Safety and Simulation Transportation Research Board, Indianapolis, USA.

Cafiso, S. e Silvestro, G. D. (2011) Performance of Safety Indicators in Identification of Black Spots on Two-Lane Rural Roads Transportation Research Record: Journal of the Transportation Research Board, 2237, 78-87. DOI: 10.3141/2237-09

Chen, Y., Persaud, B. e Sacchi, E. (2012) Improving Transferability of Safety Performance Functions by Bayesian Model Averaging. Transportation Research Record: Journal of the Transportation Research Board, 2280, 162172. DOI: $10.3141 / 2280-18$

Cheng, W. e Washington, S. (2008) New Criteria for Evaluating Methods of Identifying Hot Spots. Transportation Research Record: Journal of the Transportation Research Board, 2083, 76-85. DOI: 10.3141/2083-09

Coll, B., Moutari, S. e Marshall, A. H. (2013) Hotspots identification and ranking for road safety improvement: An alternative approach. Accident Analysis \& Prevention, 59, 604-617. DOI: 10.1016/j.aap.2013.07.012

Couto, A. e Ferreira, S. (2013) Método Probabilístico para Identificação de Zonas de Acumulação de Acidentes. Confederação Nacional do Transporte, Brasilia, Brasil. ISBN - 978-85-99082-18-8

Elvik, R. (2008) Comparative Analysis of Techniques for Identifying Locations of Hazardous Roads. Transportation Research Record: Journal of the Transportation Research Board, 2083, 72-75. DOI: 10.3141/2083-08

Ferreira, S. e Couto, A. (2012) A Probabilistic Methodology for Hot Spot Identification. TRB 2012 Annual Meeting, Transportation Research Board, Washington DC, USA, 16 pages.

Ferreira, S. e Couto, A. (2013) Hot-spot Identification: a Categorical Binary Model Approach. Transportation Research Record: Journal of the Transportation Research Board, 2386, 1-6. DOI: 10.3141/2386-01

Geedipally, S. R. e Lord, D. (2010) Identifying Hot Spots by Modeling Single-Vehicle and Multivehicle Crashes Separately Transportation Research Record: Journal of the Transportation Research Board, 2147, 97-104. DOI: 10.3141/2147-12

Geurts, K., Wets, G., Brijs, T. e Vanhoof, K. (2004) Identification and Ranking of Blackspots: Sensitivity Analysis Transportation Research Record: Journal of the Transportation Research Board, 1897, 34-42. DOI: 10.3141/189705

Hauer, E., Allery, B., Kononov, J. e Griffith, M. (2004) How best to rank sites with promise. Transportation Research 
Record: Journal of the Transportation Research Board, 1897, 48-54. DOI:10.3141/1897-07

Kononov, J. (2002) Identifying Locations with Potential for Accident Reductions: Use of Direct Diagnostics and Pattern Recognition Methodologies. Transportation Research Record: Journal of the Transportation Research Board, 1784, 153-158. DOI: 10.3141/1784-19

Lan, B. e Persaud, B. (2011) Fully Bayesian Approach to Investigate and Evaluate Ranking Criteria for BlackSpot Identification. Transportation Research Record: Journal of the Transportation Research Board, 2237, 117-125. DOI: $10.3141 / 2237-13$

Martins, J. C. (2013) Seleção de Interseções com Potencial de Redução da Sinistralidade - Aplicação do HSM. Master degree, Universidade do Porto.
Montella, A. (2010) A comparative analysis of hotspot identification methods. Accident Analysis and Prevention, 42, 571-581. DOI: 10.1016/j.aap.2009.09.025

Persaud, B., Lord, D. e Palminaso, J. (2002) Calibration and Transferability in developing accident prediction models for urban intersections. Transportation Research Record: Journal of the Transportation Research Board, 1784, 57-64. DOI: 10.3141/1784-08

Sacchi, E., Persaud, B. e Bassani, M. (2012) Assessing International Transferability of Highway Safety Manual Crash Prediction Algorithm and Its Components Transportation Research Record: Journal of the Transportation Research Board, 2279, 90-98. DOI: 10.3141/2279-11

Sawalha, Z. e Sayed, T. (2006) Transferability of accident prediction models. Safety Science, 44, 209-219. DOI: 10.1016/j.ssci.2005.09.001 